\title{
1 Mobile Elements in the Human Genome: Implications for Disease
}

2 Benoît Chénais, Le Mans Université, Le Mans, France

3

4

5

\section{Abstract}

Transposable elements (TEs) are mobile DNA sequences that represent a great portion of the human genome. TEs are divided into retrotransposons, the more abundant, and DNA transposon, which differ by their structure and transposition mechanisms. The major LINE-1 and Alu elements represent about one third of the human genome and are responsible for several chromosomal rearrangements, gene deletion, insertional mutagenesis as well as RNA splicing alteration and epigenetic regulation. DNA transposons and human endogenous retroviruses are also a source of genetic alterations. Consequently, TE insertion and mobility lead to human diseases including (but not limited to) cancer, hematologic disease, metabolic disease, neurodegenerescence, neurologic and psychiatric diseases. The herein presented examples of TE-related diseases aim to highlight the interest of increasing our knowledge of TEs through extensive genomic approaches, which may lead to new potential diagnostic markers of diseases.

Key words: Transposable elements, transposon, retrotransposon, human disease, chromosome rearrangement, gene recombination, cancer, neurologic disease, metabolic disease, genetic disease

\section{Key concepts}

- Transposable element are numerous in the human genome and the most abundant Alu and LINE-1 retro-elements represent about one third of the human genome

- Transposable elements are source of genomic rearrangements that may lead to several human diseases

- Gene expression may be affected by transposable elements through the modification of regulatory elements and epigenetic control 
- Examples of human disease related to transposable element include (but are not limited to) cancer, hematologic, metabolic and neurologic diseases

\section{Introduction: Mobile elements in the human genome}

Mobile elements or transposable elements (TEs) represent a substantial fraction of the human genome, from $45 \%$ according to the most common data (Lander et al., 2001) and even up to $66 \%$ according to further analysis (de Koning et al., 2011). The two major characteristics of these DNA sequences are their mobility in a given genome and across different genomes and their ability to increase in number in the genome, both leading to an important role in genome plasticity and evolution. TEs are diverse in nature and two main classes are identified on the basis of their mechanism of transposition: class-I (retro-) elements, or retrotransposons, use a reverse transcriptase enzyme to copy an RNA transcript into the host DNA, whereas class-II elements, or DNA transposons, mainly move through a "cut and paste" mechanism involving the excision and reinsertion of the DNA sequence of the element. In addition, a large number of repeated sequences are related to TEs but nonautonomous, i.e. dependent of the enzyme(s) encoded by a helper element, which is an autonomous TE, in order to ensure their mobility and amplification in the genome. The short interspersed nuclear elements (SINEs) are the leader class of such non-autonomous TEs that have greatly invaded the human genome (de Koning et al., 2011). See also: $\underline{D O I}$ : 10.1002/9780470015902.a0001899.pub3; DOI: 10.1002/9780470015902.a0006126.pub3 By far the most successful type of TEs in the human genome is class-I elements, which is subdivided into Long Terminal Repeats (LTR) and non-LTR elements. The latter are very predominant and $76 \%$ of human TEs are non-LTR elements, which represent at least $33.6 \%$ of the whole human genome (Lander et al., 2001) (Fig. 1). On its own, the long interspersed element (LINE)-1 accounts for 38\% of the human TEs and $16.9 \%$ of the entire genome (Fig. 1), followed by the non-autonomous SINEs, particularly the Alu repeats, which account for $24 \%$ of TEs and $10.6 \%$ of the genome (de Koning et al., 2011, Lander et al., 2001). Other SINEs found in humans are MIR and MIR3 elements. The LTR-elements are less present in the human genome and mainly represented by endogenous retroviruses (HERVs, including HERV-I, HERV-K, HERV-L) that resemble retroviruses in both their structure and mobility 
59 mechanism, but lacking a functional envelope gene. See also: DOI: 10.1002/9780470015902.a0026264; DOI: 10.1002/9780470015902.a0026711

Class-II elements, or DNA transposons, are present in small amounts in the human genome, accounting for only $6 \%$ of TEs and $2.8 \%$ of the entire genome (Fig. 1). They mainly belong to the TC-1/mariner superfamily (i.e. mariner, MER2-Tigger, Tc2), hATsuperfamily (i.e. MER-1Charlie, Zaphod), and some PiggyBac-like elements (Lander et al., 2001). See also: DOl: 10.1002/9780470015902.a0026264; DOI: 10.1002/9780470015902.a0020996.pub2 Long been considered as junk DNA or parasite DNA, TEs are now accepted as an evolutionary force that contributes to the genetic diversity of organisms. TE could be recruited on behalf of the genome to become a new gene and such TE exaptation or domestication is a source of genetic innovation, but the transposition activity may also contribute to human disorders through chromosome rearrangements, insertional mutagenesis, deregulation of gene expression, and epigenetic changes (Chénais, 2015). See also: DOl: 10.1002/9780470015902.a0026265; DOI: 10.1002/9780470015902.a0020996.pub2

\section{Mechanisms of TE's impact on human genome}

Several mechanisms are involved to explain the impact of TE on the genome and gene expression (Chénais, 2015). Genome rearrangements are favoured by the repetition of TE in the genome and the abundance of LINE-1 and Alu repeats (i.e. about $17 \%$ and $11 \%$ of the human genome, respectively) promotes ectopic recombination, leading to gene deletions, duplications and chromosome translocations or inversions (Table 1). In addition, LINE-1 and Alu elements are frequently observed within or near the breakage points of chromosomal rearrangement and LINE-1 alone account for 19\% of the 2081 breakpoints junction analysed in a dataset of 17 whole human genomes (Kidd et al., 2010). Although less present in the human genome, DNA transposons may also be a source of chromosome rearrangement as shown by a study conducted on ten types of hematolymphoid neoplasms (Rodic et al., 2013). This work showed that most translocations have no propensity to involve genomic repeats, but $47 \%$ of the translocations at the TCF3 locus on chromosome 19 involve a MER2O DNA transposon (Rodic et al., 2013). See also: DOl: 10.1002/9780470015902.a0005493.pub2; 이: 10.1002/9780470015902.a0020996.pub2 
The second way in which TEs may impact the human genome and induce diseases is through the modification of gene structure and regulatory regions (Table 2). This include the creation of new polyadenylation sites (Table 3), new exons (also called "exonisation") and new introns (or "intronisation") as well as alternative splicing modifications, e.g. exon skipping and intron retention (Table 2, Fig. 2) (Chénais, 2015). The creation of a new exon causes a frameshift or creates premature stop codons in about $79 \%$ of cases, resulting in nonfunctional sequences, which are often alternatively spliced. However, exonisation events following LINE-1 or Alu insertion may lead to human diseases (Table 2) (reviewed in Chénais, 2015). Using the LTR of HERV as promoter/enhancer is also a way to modify gene expression. This was observed, for example, in the case of the MID1 gene, associated with the X-linked form of Opitz syndrome, which transcript is fused to HERV-E LTR acting as a tissue-specific promoter/enhancer (Landry et al., 2002). See also: DOI: 10.1002/9780470015902.a0005493.pub2; DOI: 10.1002/9780470015902.a0020996.pub2; DOI: 10.1002/9780470015902.a0022886; DOI: 10.1002/9780470015902.a0026711

The third way to modify the expression of the genome concerns the epigenetic mechanisms induced or modified by TEs. A correlation between the activity of TEs and chromatin regulation, mainly DNA methylation, has been observed and hypomethylation of DNA is a cause of TEs activation (Ross et al., 2010). The relationship between pathological markers and the methylation status of whole genome CpG islands and LINE-1 elements has been particularly studied in the case of cancers (Chénais 2015). In addition, the repression of TEs through RNA interference involved essentially endogenous small interfering RNA (endosiRNA) in somatic cells and PIWI-interacting RNA (piRNA) in the germline (Saito \& Siomi, 2010). Both endo-siRNA and piRNA are transcribed from TEs themselves and a priori do not disturb the expression of protein-coding genes (Saito \& Siomi, 2010). Cancer cells are known to contain significantly lower quantities of micro-RNAs, but currently, in the absence of endo-siRNA and piRNA expression pattern in pathologic cells, it is impossible to answer the question of the putative impact of TE-related regulatory RNA on cancer or other diseases. See also: DOI: 10.1002/9780470015902.a0023593; DOI: 10.1002/9780470015902.a0025688; DOI: 10.1002/9780470015902.a0026316 
121 Several examples of cancers have been reported in the literature as related to LINE or 122 SINE/Alu insertion (Table 1) (reviewed in Chénais, 2015). More recently an increased 123 insertional activity of LINE-1 and an elevated level of LINE-1 transcript were found in primary 124 tumour samples of breast invasive carcinoma, head and neck squamous cell carcinoma and 125 lung adenocarcinoma (Clayton et al., 2016). The insertion of the LINE-1 retroelement into 126 the human proto-oncogene c-MYC was reported as early as in 1988 in human breast 127 carcinoma cells (Morse et al., 1988). This study compared the structure of the c-MYC gene 128 from breast ductal adenocarcinoma and from normal breast tissue of the same patient and 129 revealed a tumour-specific rearrangement of one MYC locus and the amplification of the other MYC locus. A non-MYC sequence with near complete homology to a LINE-1 sequence was found within the second intron of the rearranged locus. In this case, the LINE-1 sequence functioned as a mobile genetic element to produce a somatic mutation (Morse et al., 1988). Another example of a LINE-1 element somatic insertion was observed in the tumor-suppressing gene APC (adenomatous polyposis coli) from several patients with a colon cancer and also in association with desmoid tumors (reviewed in Chénais, 2015). The $B R C A 1$ and BRCA2 genes, which are associated with breast and ovarian cancer, are also the sites of Alu insertions. For example, one of the first evidenced germline mutations was the insertion of a transcriptionally active Alu element into exon 22 of BRCA2, which resulted in alternative splicing that skipped exon 22 (reviewed in Chénais, 2015). Finally, Alu insertions in the von Hippel-Lindau (VHL) gene cause large alteration at the origin of the von HippelLindau disease, a familial autosomal-dominant syndrome, predisposing to the development 142 of benign and malignant tumors (Franke et al., 2009). See also: DOl: 143 10.1002/9780470015902.a0005493.pub2

\section{- Alu recombination leading to deletion, duplication or translocation}

146 As noted above Alu repeats account for $11 \%$ of the human genome (Fig. 1). These repeats 147 are thought to induce non-allelic homologous recombination, or ectopic recombination, at 148 the origin of several cancer diseases. Numerous deletion events have been reported at the $149 B R C A 1$ locus and in a lesser extent for the BRCA2 gene, related to the unusually high density 150 of Alu repeats (Table 1). The insertion of Alu elements in the VHL gene is also involved in Alu151 mediated deletions associated with cancer (Franke et al., 2009). Large deletions in the 
152 cadherin-1 (CDH1) gene occur in $4 \%$ of hereditary diffuse gastric cancer families (Oliveira et

153 al., 2009). These deletions involve mainly non-allelic homologous recombination in Alu

154 repeat sequences, as supported by the statistically significant over-representation of Alu 155 elements around breakpoints (Oliveira et al., 2009). Homologous recombination between 156 Alu sequences and subsequent deletion have been detected at high frequency within the 157 caspase-activated DNase (CAD) gene in 13 out of the 20 human hepatoma tissues and in 158 eight of the para-cancerous cirrhotic livers (Hsieh et al., 2005) (Table 1). The recombination 159 caused exon-3 deletion, which in turn led to exon-3 skipping or replacement with a partial 160 Alu sequence, and consequential C-terminal truncation of the CAD protein (Hsieh et al., 161 2005). These data highlight the role of Alu repeats in mediating genome instability in human 162 cancers. See also: DOI: 10.1002/9780470015902.a0005493.pub2

163 Homologous recombination between Alu elements results sometimes in gene duplication as 164 reported for the BRCA1 gene, the MLL-1 (myeloid/lymphoid or mixed-lineage leukemia) gene 165 involved in acute myeloid leukemia, and the MYB proto-oncogene the duplication of which 166 leads to T-cell acute lymphoblastic leukemia (reviewed in Chénais, 2015). Duplication of the 167 MYB locus, which encodes an essential transcription factor, is frequent in human cancers. 168 The human MYB locus is flanked by Alu repeats that are responsible for its duplication 169 through somatic homologous recombination between the flanking Alu elements on sister 170 chromatids as observed in T cell acute lymphoblastic leukemia (O'Neil et al., 2007).

171 The recombination between $A l u$ repeats is also involved in the generation of the 172 Philadelphia chromosome (i.e. translocation between chromosomes 9q34 and 22q11) 173 leading to $B C R / A B L$ gene fusion in chronic myeloid leukemia (Jeffs et al., 1998). Finally, the 174 translocation between chromosomes 5q23-31 and 18q12, which involves the TRE-2 175 oncogene (TRE-USP6, ubiquitin-specific protease-6), is due to recombination between Alu 176 elements and play an important role in Ewing sarcoma development (Onno et al., 1992).

\section{- TE-related epigenetic regulation and cancer}

179 The relation between LINE-1 activation and the methylation status of DNA has been 180 particularly investigated in the case of cancers. Indeed the frequent hypomethylation of 181 chromatin in tumour cells is regarded as a facilitator of TE mobility, and especially 182 concerning LINE-1 (Ross et al., 2010). Whole genome studies revealed the preferential LINE1831 insertion in hypomethylated DNA regions of cancer cells of different origins (Ferreira et al., 
184 2014, Tubio et al., 2014). Besides, several studies highlighted the hypomethylation of the 185 LINE-1 element itself in several types of cancers (Chénais 2015) and the transcriptome 186 analysis of chronic lymphocytic leukaemia showed that TEs are globally derepressed 187 (Ferreira et al., 2014). However Lee et al. have shown that LINE-1 and Alu insertions 188 occurred preferentially in epithelial cancer but not in blood and brain cancer (Lee et al., 189 2012). In this study the authors identified almost two hundred somatic insertions of LINE-1 190 (94.5\%), Alu (5.0\%) and HERV (0.5\%) in 43 high-coverage whole genome sequencing datasets 191 from five cancer types (i.e. colorectal, glioblastoma, multiple myeloma, ovarian and prostate 192 cancer) (Lee et al., 2012). Finally, in the case of melanoma, the LINE-1 hypomethylation is 193 accompanied by the shortened relapse-free survival of patients, and a global under194 methylation of DNA impacts the metastatic formation of primary melanomas (Ecsedi et al., 195 2013).

196 Epigenetic modification of TE's regulatory sequences can impact the expression of 197 neighbouring genes. When comparing the histone enrichment, TE neighbourhood and 198 expression divergence of human genes between normal and cancer cells, a correlation was 199 observed between the presence of TEs near genes and greater changes in histone 200 enrichment (Grégoire et al., 2016).

201

202 TEs in hematologic, metabolic and/or genetic diseases

\section{- Alu-mediated deletion causing human diseases}

204 In addition to cancer, several human diseases can be attributed to Alu-mediated 205 rearrangements (Table 1). Deletions involving Alu repeats account for numerous mutations 206 in several other genes, among which the apolipoprotein $B(A P O B)$ gene, the LDL receptor $207(L D L R)$ gene, and the $\alpha$-globin and $\beta$-globin gene clusters. In a patient with homozygous 208 hypobetalipoproteinemia, the deletion of exon 21 of $A P O B$ is the consequence of a crossover 209 event between the Alu sequences in introns 20 and 21 resulting in nonreciprocal exchange between two chromosomes (Huang et al., 1989). The LDLR gene is also a place of large

211 deletions (or duplications) through homologous recombination between Alu repetitive 212 elements, which are numerous within almost all introns and in the 3'-UTR (reviewed in 213 (Hobbs et al., 1992). For example, the analysis of autosomal dominant hypercholesterolemia 214 in two Tunisian families showed the presence of a large deletion in the LDLR gene due to 
215 intrachromatid non-allelic homologous recombination between two homologous Alu 216 sequences located apart from splice sites in intronic sequences (Jelassi et al., 2012).

217 The reported TE-mediated rearrangements in the $\beta$-globin gene cluster include $\gamma^{-}, \delta$ - and $\beta$ 218 globin genes (Harteveld et al., 1997, Yu et al., 2014). For example, in an individual with 219 hereditary persistence of foetal haemoglobin, the $\beta$-globin cluster displays a large deletion of $22048.5 \mathrm{~kb}$ downstream of the $\beta$-globin gene and the $5^{\prime}$ deletion breakpoint occurs in an Alu 221 element $3^{\prime}$ to the Ay-globin gene. However, in this particular case, the recombination 222 mechanism involve non-homologous recombination since the 3' breakpoint was located 223 within a region that contains various repeats including a portion of a LINE-1 repeat, a perfect 224 160-bp palindrome, and a set of 41-bp direct repeats also present elsewhere in the human 225 genome (Henthorn et al., 1986).

226 Concerning the $\alpha$-globin gene cluster, recombination events are numerous and globally lead 227 to diverse forms of thalassemia. Following are a few examples of Alu- and/or LINE-1228 mediated recombination that lead to $\alpha$-thalassemia, an inherited hemoglobin disorder 229 characterized by a microcytic hypochromic anaemia caused by a quantitative reduction of 230 the $\alpha$-globin chain. In a Chinese family with $\alpha$-thalassemia patients, a $28.5 \mathrm{~kb}$ deletion 231 involving Alu repeats has been described that eliminated one of the duplicated $\alpha$-globin 232 genes (Yu et al., 2014). A 33-kb $\alpha^{0}$-thalassaemia deletion encompassing the $\alpha$ - and $\zeta$-globin 233 genes and pseudogenes has been observed in a Dutch family and this deletion appears 234 similar to a previously described one (Harteveld et al., 1997). The molecular analysis of 235 deletion breakpoints indicate that the mechanism leading to these $\alpha^{0}$-thalassaemia deletions 236 involves misalignment between the highly homologous Alu tandem repeats at both parental 237 sides, which are normally $33 \mathrm{~kb}$ apart. Moreover, comparison of breakpoint positions along 238 the Alu consensus sequence indicate the involvement of a 26-bp core sequence in two out of 239 five $\alpha^{0}$-thalassaemia deletions, suggesting that this sequence is a possible hotspot of 240 recombination (Harteveld et al., 1997). This suggests that Alu repeats could stimulate 241 recombination events not only by homologous pairing, but also by providing binding sites for 242 recombinogenic proteins (Harteveld et al., 1997). Finally a $8.2 \mathrm{~kb}$ deletion in the $\alpha$-globin 243 gene cluster was found in a Dutch family. This deletion involves both $\alpha$-globin genes in cis 244 and is caused by a non-homologous recombination event between an Alu and a LINE-1 245 element (Phylipsen et al., 2010). See also: DOI: 10.1002/9780470015902.a0005493.pub2 
248 The creation of a new exon, so called exonisation (Fig. 2A, Table 2), is involved in the 249 ornithine delta-aminotransferase (OAT) deficiency, which causes the gyrate atrophy of the choroid and retina, a progressive loss of vision. In this case, a G-to-C mutation in an Alu element present in the intron 3 of the OAT gene results in the creation of a new donor splice site and potentially to a new exon (Mitchell et al., 1991). This Alu insertion also causes premature termination, which again results in OAT deficiency (Mitchell et al., 1991). The Alumediated exonisation in the gene of the collagen type IV alpha-3 chain (COL4A3) may be at the origin of the Alport syndrome, a nephropathy (glomerulonephritis) due to a collagen IV defect (Knebelmann et al., 1995). In the same way, Alu-mediated exonisation has been reported in the glucuronidase beta (GUSB) gene that causes the Sly syndrome or type VII mucopolysaccharidosis (Vervoort et al., 1998). The LINE-1 element is also a potential source of exonisation as observed for the X-linked CYBB gene, encoding the beta chain of cytochrome B245 and involved in at least one case of chronic granulomatous disease 261 (Meischl et al., 2000). The LINE-1 insertion into intron 5 of the CYBB gene cause internal rearrangements and new splice sites that result in a highly heterogeneous splicing pattern with introduction of two LINE-1 fragments as new exons into the transcripts and concomitant skipping of exonic coding sequence (Meischl et al., 2000).

277 skipping. For example the insertion of LINE-1 induces skipping of exon 44, causing Duchenne 278 muscular dystrophy (Narita et al., 1993). Insertion of an Alu-like sequence downstream of 
intron 11 was observed in individuals with X-linked dilated cardiomyopathy (Ferlini et al.,

280 1998). The insertion induces a rearrangement that activates a cryptic splice site in intron 11 281 and produces an alternative transcript containing the Alu-like sequence and part of the 282 adjacent intron 11, spliced between exons 11 and 12, but not translated due to the presence of numerous stop codons (Ferlini et al., 1998). The LINE-1 element is responsible for a large deletion in the X-linked pyruvate dehydrogenase E1 (PDHX) gene, but the LINE-1 insertion may also lead, at last in one patient, to an aberrantly spliced isoform resulting from the use of two cryptic splice sites (Mine et al., 2007).

Finally, intron retention (Fig. 2C) may happen when an error occurs in normal splicing leading to retention of a transcript-confirmed intron within a transcript-confirmed exon and thus in the mature mRNA. Such events may be affected by TEs, as demonstrated in the case of $L I N E-1$ insertion in the CYBB gene that causes chronic granulomatous disease (Meischl et al., 2000).

- Alu and LINE-1 derived new polyadenylation sites

LINE-1 elements include a polyadenylation signal within their own sequence, and the polyadenylation signal (i.e. AATAAA sequence) is frequently created in the A-rich tails of both SINEs and LINEs. Therefore human retroelements, such as LINE-1 and Alu, may introduce intragenic polyadenylation signals creating new mRNA isoforms. Several diseases have been linked to the introduction of a new polyadenylation site through LINE-1 or Alu element insertion in human genes, such as haemophilia $A$ and $B, X$-linked dilated cardiomyopathy, autoimmune lymphoproliferative syndrome, hypo-calciuric hypercalcemia and neonatal severe hyperparathyroidism as reviewed in Chénais (2015) (Table 3). Besides, the LTR sequence of HERV is also a source of new polyadenylation signal as observed with HERV-K in the breast cancer cell line T47D (Baust et al., 2000) and with HERV$H$ in the HHLA2 and HHLA3 genes (Mager et al., 1999); but no obvious relation with a pathology was described to date.

\section{- LINE-1 and chromatin regulation}

308 As described above for cancer, the chromatin structure and/or epigenetic regulation associated with TE insertion may lead to non-cancer human diseases but examples are rare

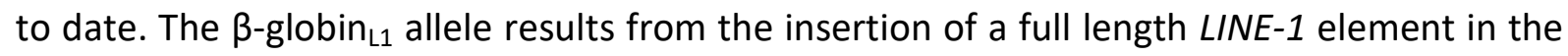


311 intron 2 of the $\beta$-globin gene. The decreased rate of transcription of this $\beta$-globin ${ }_{\llcorner 1}$ allele is 312 due to the hypermethylated profile of its promoter and enhancer sequences, which results 313 in a transcriptional down-regulation and thus the $\beta^{+}$-thalassemia phenotype (Lanikova et al., 314 2013).

\section{TE involvement in neurological diseases}

317 As for cancer, metabolic and hematologic diseases, TE insertion may be the cause of neurological diseases or syndromes. The insertion of a LINE-1 element in the ribosomal S6 kinase 2 (RSK2) gene was reported in a patient with Coffin-Lowry syndrome, a disease characterised by psychomotor and growth retardation, facial dysmorphy and squeletic abnormalities. This LINE-1 insertion at position -8 of intron 3 leads to a skipping of exon 4 and then to a reading-frame shift and the appearance of a premature stop codon (Table 2)

323 (Martinez-Garay et al., 2003).

The Fabry disease, characterized by a neurodegenerescence of the central nervous system, is an X-linked recessive disorder of glycosphingolipid catabolism, which results from the deficient activity of the lysosomal hydrolase $\alpha$-galactosidase A encoded by an Alu-rich gene, that displays 12 Alu elements within its $12 \mathrm{~kb}$ sequence (Kornreich et al., 1990). Several mutations and gene rearrangements have been described to be at the origin of the disease (Table 1), among which only one deletion was originally identified as an Alu-Alu recombination, and ten other as Alu-Alu rearangements (Kornreich et al., 1990). Thereafter,

331 a large $3.1 \mathrm{~kb}$ deletion due to Alu-Alu recombination and including the whole exon 2 was 332 identified among 50 new mutations at the origin of Fabry disease (Shabbeer et al., 2006).

333 The Sandhoff disease, a recessive lysosomal storage disease, is another example of TE334 related neurodegenerative disease (Table 1). The deficience of $\beta$-hexosaminidase (HEXB) 335 activity is due to deletion alleles of the HEXB gene in $27 \%$ of the examined dataset (Neote et al., 1990). HEXB contains two Alu repeats that are involved in the deletion event, which removes about $16 \mathrm{~kb}$ including the HEXB promoter, exons 1 to 5, and part of intron 5 . The

338 deletion also resulted in a reconstituted Alu sequence, the left half of which comes from the 5' Alu sequence and the right half of which from the 3' Alu sequence (Neote et al., 1990). 
Neurofibromatosis type I is a common genetic disease linked to the NF-1 tumor suppressor gene and characterized by changes in skin coloring and the growth of benign tumors along nerves (i.e. neurofibromas). The insertion of an Alu sequence in the intron of the NF-1 gene

343 leads to a deletion and reading frame shift in the downstream exon during splicing, which 344 could be a cause of the disease (Table 2) (Wallace et al., 1991). See also: DOI: 10.1002/9780470015902.a0005493.pub2

346 Elevated expression of TEs has been observed in several human neurodegenerative 347 disorders including sporadic Creutzfeld-Jakob disease, age-related macular degeneration and amyotrophic lateral sclerosis (ALS). For example, the RNA-binding protein TDP-43, which is involved in ALS and frontotemporal lobar degeneration (FTLD), was shown to interact with many TE transcripts and moreover this association of TDP-43 with many of its TE targets is reduced in FTLD patients (Li et al., 2012).

352 Finally, a link between TEs and psychiatric disorders was also highlighted (Guffanti et al., 353 2014). Particularly, whole-genome sequencing revealed brain specific LINE-1 insertion in 354 patients localized preferentially to synapse- and schizophrenia-related genes, suggesting a 355 causal role of LINE-1 in the onset of the disease (Bundo et al., 2014).

\section{Conclusion}

358 First considered as junk DNA, TEs are now considered as major actors of genome evolution 359 and their role in genome plasticity is now obviously demonstrated. This involves several molecular mechanisms besides TEs insertion per se, including DNA recombination and chromosome rearrangements (especially deletion), modification of gene expression through the introduction or alteration of poly-A sites, splicing sites, exons or introns and regulatory elements, and finally the domestication of TEs leading to new functionalities.

364 The high abundance of TEs in the human genome, especially Alu and LINE-1 repeats, make 365 them responsible for the molecular origin of several diseases as presented above. Therefore increasing our knowledge of TEs through extensive genomic approaches may lead to new 367 potential diagnostic markers of diseases and prenatal markers of genetic disorders. In this 368 way the recent development of combined molecular and bioinformatics tools is of great interest, such as the work by Tang et al., which have detected somatic LINE-1 insertions in ovarian cancer (Tang et al., 2017). 
372

373

374

375

376

377

378

379

380

381

382

383

384

385

386

387

388

389

390

391

392

393

394

395

396

397

398

399

400

References

Baust C, Seifarth W, Germaier H, Hehlmann R, Leib-Mosch C (2000) HERV-K-T47D-related long terminal repeats mediate polyadenylation of cellular transcripts. Genomics 66(1): 98-103.

Bundo M, Toyoshima M, Okada Y et al. (2014) Increased I1 retrotransposition in the neuronal genome in schizophrenia. Neuron 81(2): 306-313.

Chénais B (2015) Transposable elements in cancer and other human diseases. Current Cancer Drug Targets 15(3): 227-242.

Clayton EA, Wang L, Rishishwar L et al. (2016) Patterns of transposable element expression and insertion in cancer. Frontiers in Molecular Biosciences 3: 76.

de Koning AP, Gu W, Castoe TA, Batzer MA, Pollock DD (2011) Repetitive elements may comprise over two-thirds of the human genome. PLoS Genetics 7(12): e1002384.

Ecsedi SI, Hernandez-Vargas H, Lima SC et al. (2013) Transposable hypomethylation is associated with metastatic capacity of primary melanomas. International Journal of Clinical and Experimental Pathology 6(12): 2943-2948.

Ferlini A, Galie N, Merlini L et al. (1998) A novel Alu-like element rearranged in the dystrophin gene causes a splicing mutation in a family with X-linked dilated cardiomyopathy. American Journal of Human Genetics 63(2): 436-446.

Ferreira PG, Jares P, Rico D et al. (2014) Transcriptome characterization by RNA sequencing identifies a major molecular and clinical subdivision in chronic lymphocytic leukemia. Genome Research 24(2): 212-226.

Franke G, Bausch B, Hoffmann MM et al. (2009) Alu-Alu recombination underlies the vast majority of large VHL germline deletions: Molecular characterization and genotypephenotype correlations in VHL patients. Human Mutation 30(5): 776-786.

Ganguly A, Dunbar T, Chen P, Godmilow L, Ganguly T (2003) Exon skipping caused by an intronic insertion of a young Alu YB9 element leads to severe hemophilia A. Human Genetics 113(4): 348-352.

Grégoire L, Haudry A, Lerat E (2016) The transposable element environment of human genes is associated with histone and expression changes in cancer. BMC Genomics 17: 588. 
401

402

403

404

405

406

407

408

409

410

411

412

413

414

415

416

417

418

419

420

421

422

423

424

425

426

427

428

429

430

431

432

Guffanti G, Gaudi S, Fallon JH et al. (2014) Transposable elements and psychiatric disorders. American Journal of Medical Genetics, part B: Neuropsychiatric Genetics 165B(3): 201216.

Harteveld KL, Losekoot M, Fodde R, Giordano PC, Bernini LF (1997) The involvement of Alu repeats in recombination events at the alpha-globin gene cluster: Characterization of two alphazero-thalassaemia deletion breakpoints. Human Genetics 99(4): 528-534.

Henthorn PS, Mager DL, Huisman TH, Smithies O (1986) A gene deletion ending within a complex array of repeated sequences 3 ' to the human beta-globin gene cluster. Proceedings of the National Academy of Sciences of the United States of America 83(14): 5194-5198.

Hobbs HH, Brown MS, Goldstein JL (1992) Molecular genetics of the LDL receptor gene in familial hypercholesterolemia. Human Mutation 1(6): 445-466.

Hsieh SY, Chen WY, Yeh TS, Sheen IS, Huang SF (2005) High-frequency Alu-mediated genomic recombination/deletion within the caspase-activated DNase gene in human hepatoma. Oncogene 24(43): 6584-6589.

Huang LS, Ripps ME, Korman SH, Deckelbaum RJ, Breslow JL (1989)

Hypobetalipoproteinemia due to an apolipoprotein $B$ gene exon 21 deletion derived by Alu-Alu recombination. The Journal of Biological Chemistry 264(19): 11394-11400.

Jeffs AR, Benjes SM, Smith TL, Sowerby SJ, Morris CM (1998) The BCR gene recombines preferentially with Alu elements in complex $B C R-A B L$ translocations of chronic myeloid leukaemia. Human Molecular Genetics 7(5): 767-776.

Jelassi A, Slimani A, Rabes JP et al. (2012) Genomic characterization of two deletions in the $L D L R$ gene in tunisian patients with familial hypercholesterolemia. Clinica Chimica Acta 414: 146-151.

Kidd JM, Graves T, Newman TL et al. (2010) A human genome structural variation sequencing resource reveals insights into mutational mechanisms. Cell 143(5): 837-847.

Knebelmann B, Forestier L, Drouot L et al. (1995) Splice-mediated insertion of an Alu sequence in the COLAA3 mRNA causing autosomal recessive Alport syndrome. Human Molecular Genetics 4(4): 675-679.

Kornreich R, Bishop DF, Desnick RJ (1990) Alpha-galactosidase A gene rearrangements causing Fabry disease. Identification of short direct repeats at breakpoints in an Alu-rich gene. The Journal of Biological Chemistry 265(16): 9319-9326. 
433 Lander ES, Linton LM, Birren B et al. (2001) Initial sequencing and analysis of the human 434 genome. Nature 409(6822): 860-921.

435 Landry JR, Rouhi A, Medstrand P, Mager DL (2002) The Opitz syndrome gene MID1 is 436 transcribed from a human endogenous retroviral promoter. Molecular Biology and $437 \quad$ Evolution 19(11): 1934-1942.

438 Lanikova L, Kucerova J, Indrak K et al. (2013) Beta-thalassemia due to intronic LINE-1 439 insertion in the beta-globin gene (HBB): Molecular mechanisms underlying reduced 440 transcript levels of the beta-globin(L1) allele. Human Mutation 34(10): 1361-1365. 441 Lee $E$, Iskow R, Yang L et al. (2012) Landscape of somatic retrotransposition in human 442 cancers. Science 337(6097): 967-971.

Li W, Jin Y, Prazak L, Hammell M, Dubnau J (2012) Transposable elements in TDP-43mediated neurodegenerative disorders. PLoS One 7(9): e44099.

Mager DL, Hunter DG, Schertzer M, Freeman JD (1999) Endogenous retroviruses provide the 446 the MYC locus by a LINE-1 sequence in a human breast carcinoma. Nature 333(6168): 8790. 
464

465

466

467

468

469

470

471

472

473

474

475

476

477

478

479

480

481

482

483

484

485

486

487

488

489

490

491

492

493

494

495

Narita N, Nishio H, Kitoh Y et al. (1993) Insertion of a 5' truncated L1 element into the 3' end of exon 44 of the dystrophin gene resulted in skipping of the exon during splicing in a case of Duchenne muscular dystrophy. The Journal of Clinical Investigation 91(5): 18621867.

Neote K, McInnes B, Mahuran DJ, Gravel RA (1990) Structure and distribution of an Alu-type deletion mutation in Sandhoff disease. The Journal of Clinical Investigation 86(5): 15241531.

O'Neil J, Tchinda J, Gutierrez A et al. (2007) Alu elements mediate MYB gene tandem duplication in human T-ALL. The Journal of Experimental Medicine 204(13): 3059-3066.

Oldridge M, Zackai EH, McDonald-McGinn DM et al. (1999) De novo Alu-element insertions in FGFR2 identify a distinct pathological basis for Apert syndrome. American Journal of Human Genetics 64(2): 446-461.

Oliveira C, Senz J, Kaurah P et al. (2009) Germline CDH1 deletions in hereditary diffuse gastric cancer families. Human Molecular Genetics 18(9): 1545-1555.

Onno M, Nakamura T, Hillova J, Hill M (1992) Rearrangement of the human tre oncogene by homologous recombination between Alu repeats of nucleotide sequences from two different chromosomes. Oncogene 7(12): 2519-2523.

Phylipsen M, Vogelaar IP, Schaap RA et al. (2010) A new alpha(0)-thalassemia deletion found in a Dutch family (--(aw)). Blood Cells Molecular Disease 45(2): 133-135.

Rodic N, Zampella JG, Cornish TC, Wheelan SJ, Burns KH (2013) Translocation junctions in TCF3-PBX1 acute lymphoblastic leukemia/lymphoma cluster near transposable elements. Mobile DNA 4(1): 22.

Ross JP, Rand KN, Molloy PL (2010) Hypomethylation of repeated DNA sequences in cancer. Epigenomics 2(2): 245-269.

Saito K, Siomi MC (2010) Small RNA-mediated quiescence of transposable elements in animals. Developmental Cell 19(5): 687-697.

Shabbeer J, Yasuda M, Benson SD, Desnick RJ (2006) Fabry disease: Identification of 50 novel alpha-galactosidase a mutations causing the classic phenotype and three-dimensional structural analysis of 29 missense mutations. Human Genomics 2(5): 297-309.

Tang Z, Steranka JP, Ma S et al. (2017) Human transposon insertion profiling: Analysis, visualization and identification of somatic line-1 insertions in ovarian cancer. Proceedings of the National Academy of Sciences of the United States of America 114(5): E733-E740. 
496

497

498

499

500

501

502

503

504

505

506

507

508

509

510

511

512

513

514

515

516

517

518

519

520

521

522

523

524

525

526

Tubio JM, Li Y, Ju YS et al. (2014) Mobile DNA in cancer. Extensive transduction of nonrepetitive DNA mediated by L1 retrotransposition in cancer genomes. Science 345(6196): 1251343.

Vervoort R, Gitzelmann R, Lissens W, Liebaers I (1998) A mutation (IVS8+0.6kbdeltc) creating a new donor splice site activates a cryptic exon in an alu-element in intron 8 of the human beta-glucuronidase gene. Human Genetics 103(6): 686-693.

Wallace MR, Andersen LB, Saulino AM et al. (1991) A de novo Alu insertion results in neurofibromatosis type 1. Nature 353(6347): 864-866.

Yu J, Xie J, Luo L, Li Z (2014) An Alu element-mediated 28.5 kb alpha-thalassemia deletion found in a chinese family. Hemoglobin 38(6): 427-430.

\section{Further readings}

Chénais B, Caruso A, Hiard S, Casse N (2012) The impact of transposable elements on eukaryotic genomes: From genome size increase to genetic adaptation to stressful environments. Gene 509(1): 7-15.

Chénais B (2013) Transposable elements and human cancer: A causal relationship? Biochimica et Biophysica Acta 1835(1): 28-35.

Cordaux R, Batzer MA (2009) The impact of retrotransposons on human genome evolution. Nature Reviews Genetics 10(10): 691-703.

Feschotte C, Pritham EJ (2007) DNA transposons and the evolution of eukaryotic genomes. Annual Review of Genetics 41: 331-368.

Kulis M, Queiros AC, Beekman R, Martin-Subero Jl (2013) Intragenic DNA methylation in transcriptional regulation, normal differentiation and cancer. Biochimica et Biophysica Acta 1829(11): 1161-1174.

Levin HL, Moran JV (2011) Dynamic interactions between transposable elements and their hosts. Nature Reviews Genetics 12(9): 615-627.

Mazoyer S (2005) Genomic rearrangements in the BRCA1 and BRCA2 genes. Human Mutation 25(5): 415-422.

Reilly MT, Faulkner GJ, Dubnau J, Ponomarev I, Gage FH (2013) The role of transposable elements in health and diseases of the central nervous system. Journal of Neuroscience 33(45): 17577-17586. 
Roy-Engel AM, El-Sawy M, Farooq L et al. (2005) Human retroelements may introduce intragenic polyadenylation signals. Cytogenetics and Genome Research 110(1-4): 365371.

Sinzelle L, Izsvak Z, Ivics Z (2009) Molecular domestication of transposable elements: From detrimental parasites to useful host genes. Cellular and Molecular Life Sciences 66(6): 1073-1093.

\section{Glossary}

Ectopic recombination: aberrant form of DNA recombination between non-homologous loci, rather than along homologous loci. Such recombination is often driven by transposable element and results in dramatic chromosomal rearrangement, which is generally deleterious.

Exonisation: recruiting of a new exon from non-protein-coding, such as intronic DNA sequences or transposable elements.

Exon skipping: splicing modification following the insertion of a transposable element that result in the skipping of one or more exon during mRNA maturation.

Intronisation: creation of new intron following the insertion of a transposable element sequence.

Retrotransposon or retroelement: transposable element that needs to be retro-transcribed from RNA before reinsertion in the genomic DNA. The structure of retrotransposons may be more or less complex and could include, or not, long terminal repeat (LTR) sequences. Transposon: in eukaryotes, sequence of repeated DNA that encodes the transposase enzyme sufficient to assume the mobility of the transposable element. 
Table 1. Examples of human diseases linked to TE insertion, TE-mediated deletions or TEmediated chromosomal rearrangements.

\begin{tabular}{|c|c|c|c|}
\hline Gene & Type of TE & Type of event & Disease or disorder \\
\hline$A P C$ & LINE-1 & insertion & Colon cancer, desmoid tumours \\
\hline$A P O B$ & Alu & deletion & Hypo beta lipoproteinemia \\
\hline$B C R-A B L$ & Alu & $\begin{array}{l}\text { translocation } \\
\mathrm{t}(9 ; 22)(\mathrm{q} 34 ; \mathrm{q} 11)\end{array}$ & Chronic myeloid leukemia \\
\hline BRCA1 & Alu & $\begin{array}{l}\text { insertion, deletion } \\
\text { and duplication }\end{array}$ & Breast/ovarian cancer \\
\hline$B R C A 2$ & Alu & insertion, duplication & Breast/ovarian cancer \\
\hline$C A D$ & Alu & deletion & Hepatoma \\
\hline $\mathrm{CDH} 1$ & Alu & deletion & Hereditary diffuse gastric cancer \\
\hline EWSR1-ETV & Alu & $\begin{array}{l}\text { translocation } \\
\mathrm{t}(5 \mathrm{q} 23 \mathrm{q} 31)(18 \mathrm{q} 12)\end{array}$ & Ewing sarcoma \\
\hline$a-G A L A$ & Alu & deletion & Fabry disease \\
\hline$H B B$ & Alu & deletion & Thalassemia \\
\hline$H E X B$ & Alu & deletion & Sandhoff disease \\
\hline$L D L R$ & Alu & deletion & Familial hyper-cholesterolemia \\
\hline NF1 & Alu & insertion & Neurofibroma \\
\hline MLL1 & Alu & duplication & Acute myeloid leukemia \\
\hline MYB & Alu & duplication & T-cell lymphoblastic leukemia \\
\hline MYC & LINE-1 & insertion & Breast carcinoma \\
\hline TCF3 & MER20 & translocation & hematolymphoid neoplasm \\
\hline$V H L$ & Alu & deletion & Von Hippel Lindau disease \\
\hline
\end{tabular}


Table 2. Examples of TE-mediated alterations of mRNA splicing at the origin of human diseases, including exonisation, exon skipping, intron retention and aberrant splicing.

\begin{tabular}{|c|c|c|c|}
\hline Gene & Type of TE & Type of event $^{a}$ & Disease or disorder \\
\hline$B R C A 2$ & Alu & aberrant splicing & Breast cancer \\
\hline COL4A3 & Alu & exonisation & Alport syndrome \\
\hline$C Y B B$ & LINE-1 & $\begin{array}{l}\text { exonisation, } \\
\text { intron retention }\end{array}$ & Chronic granulomatous disease \\
\hline$D M D$ & LINE-1 & exon skipping & Duchenne Muscular Dystrophy \\
\hline$D M D$ & Alu & aberrant splicing & X-linked dilated cardiomyopathy \\
\hline F8 & Alu & exon skipping & Hemophilia A \\
\hline FAS & Alu & exon skipping & $\begin{array}{l}\text { Autoimmune lymphoproliferative } \\
\text { syndrome }\end{array}$ \\
\hline FGFR2 & Alu & $\begin{array}{l}\text { exon skipping, } \\
\text { aberrant splicing }\end{array}$ & Apert syndrome \\
\hline FKTN & LINE-1 & exon skipping & $\begin{array}{l}\text { Fukuyama-type congenital muscular } \\
\text { dystrophy }\end{array}$ \\
\hline GUSB & Alu & exonisation & Sly syndrome \\
\hline IDS & Alu & exon skipping & Hunter syndrome \\
\hline MID1 & HERV-E & $\begin{array}{l}\text { new regulatory } \\
\text { region }\end{array}$ & Opitz syndrome \\
\hline NF1 & Alu & $\begin{array}{l}\text { aberant splicing, } \\
\text { reading frame shift }\end{array}$ & Neurofibromatosis type 1 \\
\hline OAT & Alu & exonisation & $\begin{array}{l}\text { ornithine delta-aminotransferase } \\
\text { deficiency }\end{array}$ \\
\hline OPA1 & Alu & exon skipping & Autosomal dominant optic atrophy \\
\hline PDHX & LINE-1 & aberrant splicing & $\begin{array}{l}\text { Pyruvate dehydrogenase complex } \\
\text { deficiency }\end{array}$ \\
\hline$R P 2$ & LINE-1 & $\begin{array}{l}\text { aberrant splicing, } \\
\text { reading frame shift }\end{array}$ & $\mathrm{X}$-linked retinitis pigmentosa \\
\hline RSK2 & LINE-1 & exon skipping & Coffin-Lowry syndrome \\
\hline
\end{tabular}


Table 3. Examples of TE-mediated creation of new polyadenyation site involved in the onset of human diseases.

\begin{tabular}{ccl}
\hline Gene & Type of TE & \multicolumn{1}{c}{ Disease or disorder } \\
\hline APC & LINE-1 & Colon cancer \\
ASR & Alu & $\begin{array}{l}\text { Hypocalciuric hypercalcemia and neonatal } \\
\text { severe hyper-parathyroidism }\end{array}$ \\
DMD & LINE-1 & $\begin{array}{l}\text { X-linked dilated cardiomyopathy } \\
\text { F9 }\end{array}$ \\
F9 & AIU & Hemophilia B \\
FAS & Alu & Autoimmune lympho-proliferative syndrome \\
HHLA2, 3 & HERV-H & unkwown \\
\hline
\end{tabular}




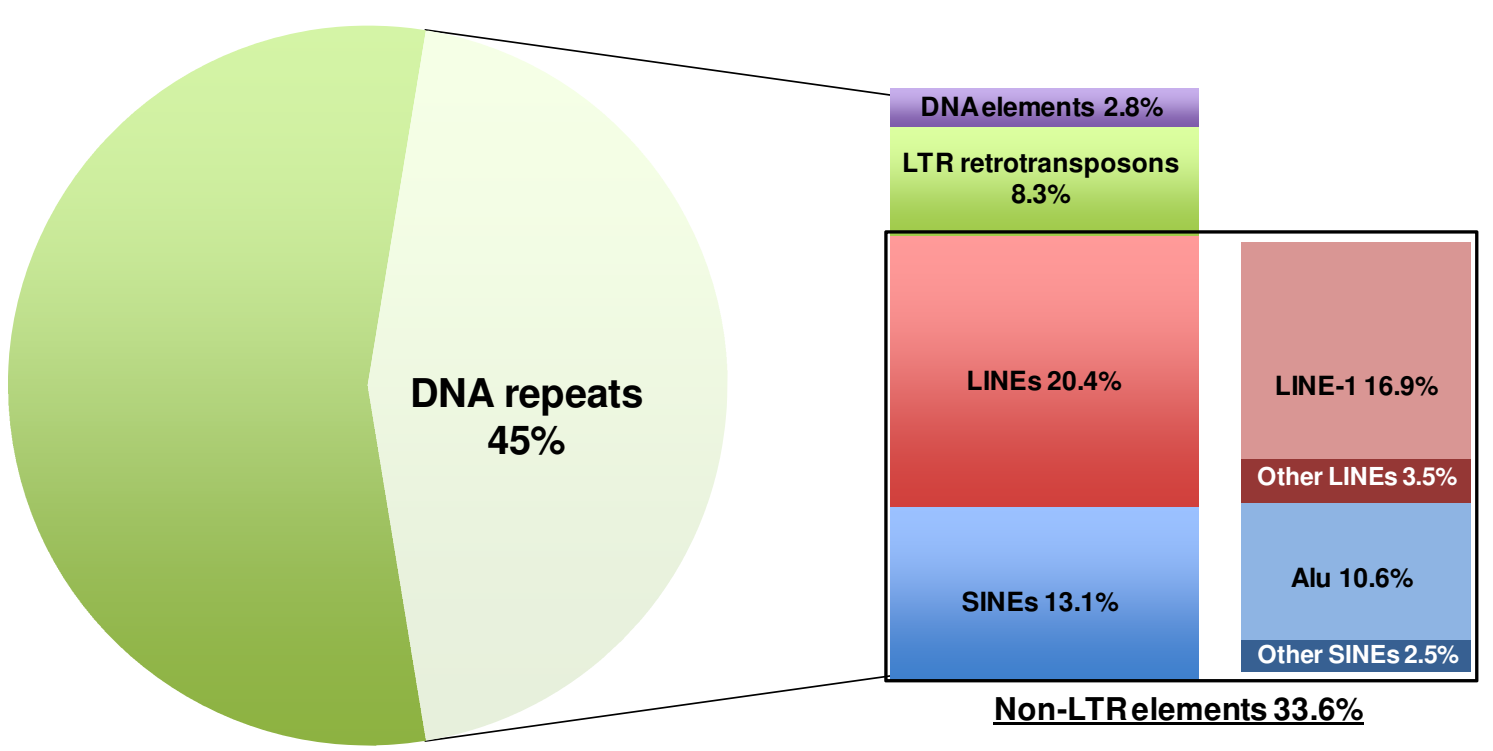

Figure 1. Proportion of TEs in the human genome (Chénais, Current Cancer Drug Targets 2015). The percentage of each class or sub-class of TE is indicated with respect to the whole genome according to data from Lander et al. (2001). 


\section{A) Exonisation}

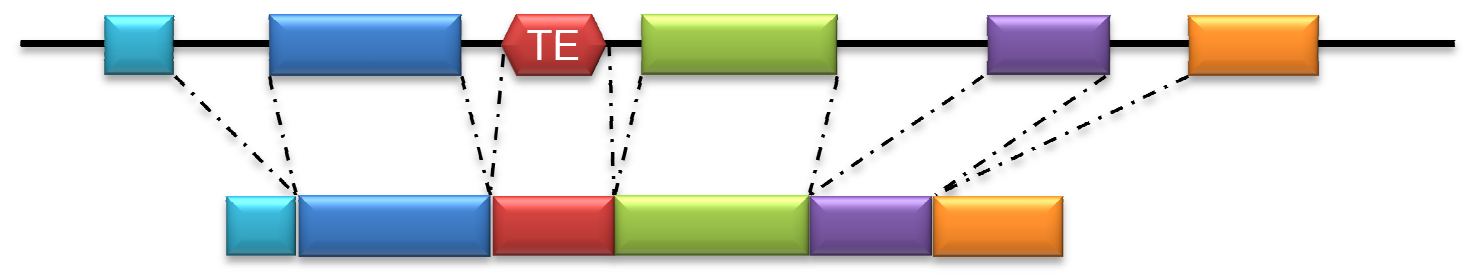

$\rightarrow$ New protein isoform or mRNA degradation

\section{B) Exon skipping}

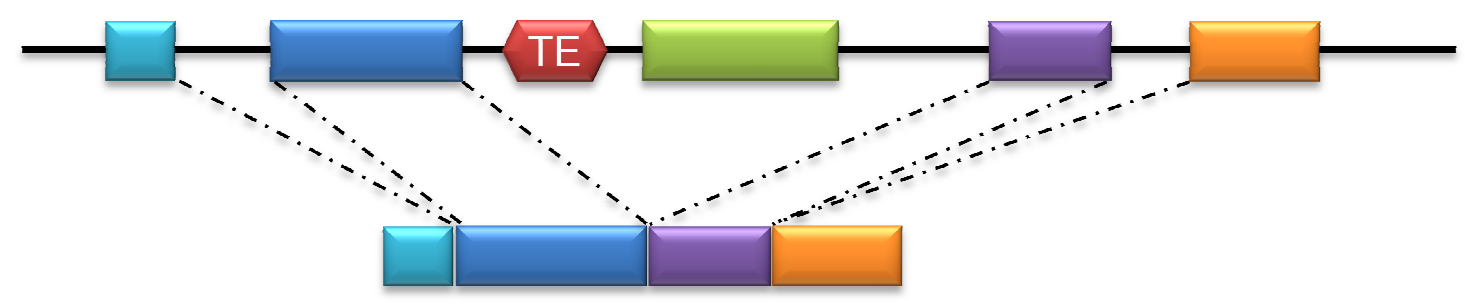

$\rightarrow$ Truncated protein

\section{C) Intron retention}

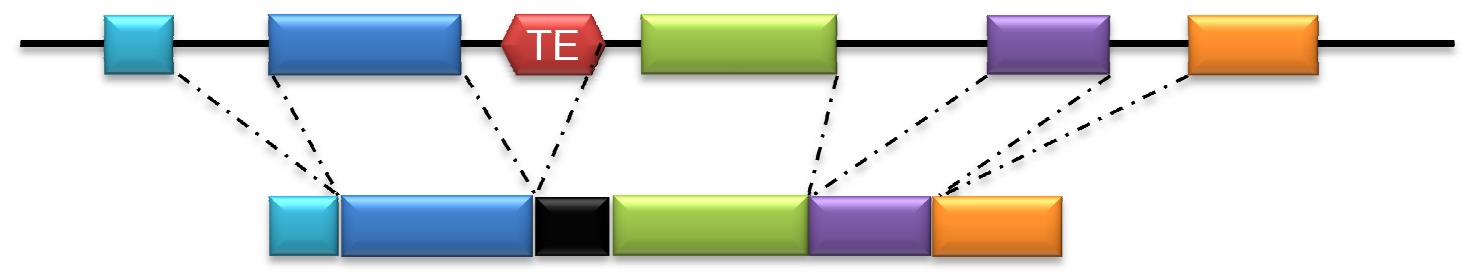

$\rightarrow$ Aberrant protein or mRNA degradation

Figure 2. Simplified view of the main consequences of TE insertion on mRNA splicing. A) exonisation; B) exon skipping; C) intron retention. Red box figure the TE and colored boxes correspond to exons; introns are represented as black lines; dashed lines indicate the splicing events. (Chénais, Current Cancer Drug Targets 2015). 